\title{
Sleep and the Time Course of Motor Skill Learning
}

\author{
Matthew P. Walker, ${ }^{1}$ Tiffany Brakefield, Joshua Seidman, Alexandra Morgan, \\ J. Allan Hobson, and Robert Stickgold \\ Laboratory of Neurophysiology, Massachusetts Mental Health Center, Department of Psychiatry, Harvard Medical School, \\ Boston, Massachusetts 02115, USA
}

\begin{abstract}
Growing evidence suggests that sleep plays an important role in the process of procedural learning. Most recently, sleep has been implicated in the continued development of motor-skill learning following initial acquisition. However, the temporal evolution of motor learning before and after sleep, the effects of different training regimens, and the long-term development of motor learning across multiple nights of sleep remain unknown. Here, we report data for subjects trained and retested on a sequential finger-tapping task across multiple days. The findings demonstrate firstly that following initial training, small practice-dependent improvements are possible before, but not following the large practice-independent gains that develop across a night of sleep. Secondly, doubling the quantity of initial training does not alter the amount of subsequent sleep-dependent learning that develops overnight. Thirdly, the amount of sleep-dependent learning does not correlate with the amount of practice-dependent learning achieved during training, suggesting the existence of two discrete motor-learning processes. Finally, whereas the majority of sleep-dependent motor-skill learning develops during the first night of sleep following training, additional nights of sleep still offer continued improvements.
\end{abstract}

Evidence from animal and human studies continues to suggest that the brain-state of sleep plays an important role in the process of memory formation. In humans, sleep has been most consistently implicated in the development of procedural learning (i.e., perceptual and motor skills), specifically in the ongoing process of memory consolidation, following the initial stage of memory acquisition (Karni et al. 1994; Gais et al. 2000; Stickgold et al. 2000a,b; Walker et al. 2002).

Several different factors are considered to be important in procedural memory formation. One critical element is the amount of training or practice during task acquisition, which strongly influences both behavioral learning (Karni et al. 1995; Karni and Bertini 1997; Rattoni and Escobar 2000) and functional brain changes (Karni et al. 1995; Jancke et al. 2001). It is widely accepted that, following effective acquisition, a specific memory representation is formed, which can then undergo further modification during the process of consolidation.

The process of consolidation was initially believed to evolve in a time-dependent manner (Brashers-Krug et al. 1996; Karni et al. 1998; McGaugh 2000). However, this concept has been refined recently, with several studies suggesting that, in addition to sufficient practice during acquisition (which is usually accompanied by rapid learning within the session), posttraining consolidation offers a second, delayed stage of learning that develops in the absence of further training. But instead of evolving simply as a function of time per se, this delayed improvement develops exclusively across periods of sleep and not wake for certain forms of learning (Karni et al. 1994; Gais et al. 2000; Stickgold et al. 2000a,b; Fischer et al. 2002; Walker et al. 2002).

\footnotetext{
${ }^{1}$ Corresponding author.

E-MAIL mwalker@hms.harvard.edu; FAX (617) 734-7851.

Article and publication are at http://www.learnmem.org/cgi/doi/10.1101/ $\operatorname{lm} .58503$.
}

For example, in the visual domain, Karni et al. (1994) have demonstrated that, following training, delayed learning of a texture discrimination task occurs during a night of sleep, and that selective disruption of rapid eye movement (REM) sleep prevents this overnight performance gain. Using the same task, Stickgold and colleagues have shown firstly that learning of this task does not benefit from periods of 3-12 h of wake following the acquisition stage of memory formation (Stickgold et al. 2000b), whereas sleep triggers significant additional performance improvements, with the overnight benefits correlating strongly with the amount of slow-wave sleep (SWS; combined stage-III and stage-IV NREM sleep) early in the night, as well as the amount of REM sleep late in the night (Stickgold et al. 2000b). Stickgold and colleagues have also demonstrated that delayed performance benefits are absolutely dependent on the first night of sleep after acquisition (Stickgold et al. 2000a). Also following training on this same visual skill task, Gais et al. (2000) selectively deprived subjects of sleep early in the night (rich in SWS) or late in the night (rich in REM sleep), and concluded that enhanced memory consolidation is triggered by SWS-related processes, whereas REM sleep promotes additional consolidation, but only after periods of SWS sleep (Gais et al. 2000).

Several lines of evidence have also identified delayed motorskill learning that is sleep, and not just time dependent. First, Walker et al. (2002), have described evidence of sleep-dependent learning in the motor system using a sequential finger-tapping task. Subjects were trained either in the morning or evening and retested at subsequent 12-h intervals following wake or sleep. Although practice on the motor-skill task improved performance within the training session for all groups equally, regardless of time of day, subjects trained in the morning demonstrated no significant improvement in speed when retested after $12 \mathrm{~h}$ of wake. In contrast, they showed an average $20 \%$ improvement by the next morning, following a night of sleep. Subjects who were 
instead trained in the evening, demonstrated large and significant improvements in performance overnight, just 12-h posttraining, but showed no significant additional learning after a further $12 \mathrm{~h}$ of wake. Thus, significant delayed improvement was only seen across a night of sleep and not over a similar period of wake, regardless of whether the time awake or time asleep came first. Second, when the degree of overnight improvement in motor learning was correlated with sleep recordings, a significant positive correlation was found with the percentage of stage-II NREM sleep, particularly late in the night, further implicating sleep in the observed learning effect. Third, similar data by Fischer et al. (2002) have recently confirmed the findings of Walker et al. (2002), with the additional evidence that, as with visual skill learning (Stickgold et al. 2000a), sleep on the first night following training is critical for the delayed improvement to develop, and that sleep during the day triggers similar improvements to those achieved following nocturnal sleep.

Thus, practice clearly improves performance within a session on certain tasks, whereas sleep triggers an additional consolidation phase in the absence of further task engagement. Building on these previous findings, we now describe the nature and time course of this procedural motor-skill learning, and ex- amine the relationship between practice-dependent and delayed sleep-dependent motor learning.

Four specific questions are addressed in this study: (1) After an initial training session, are there differences in the profile of motor skill learning across the first $12 \mathrm{~h}$ of wake prior to a night of sleep compared with the profile of motor-skill learning across the $12 \mathrm{~h}$ of wake following a subsequent night of sleep? (2) How does a second full training session affect subsequent overnight sleep-dependent learning? (3) How does improvement after three nights of sleep compare with one night of sleep? (4) Is the amount of delayed, sleep-dependent learning correlated with the earlier, rapid, practice-dependent learning?

\section{RESULTS}

\section{Practice-Dependent Learning During Training}

The forty subjects in groups 1-4 were trained on the finger-skill task at either $10 \mathrm{AM}$ or $1 \mathrm{PM}$ on day 1 , with the learning curves for each group displayed in Figure 1.

\section{Training Session 1}

Improvement in performance across the first 12-trial training session (Session One) was similar for all groups, with no significant

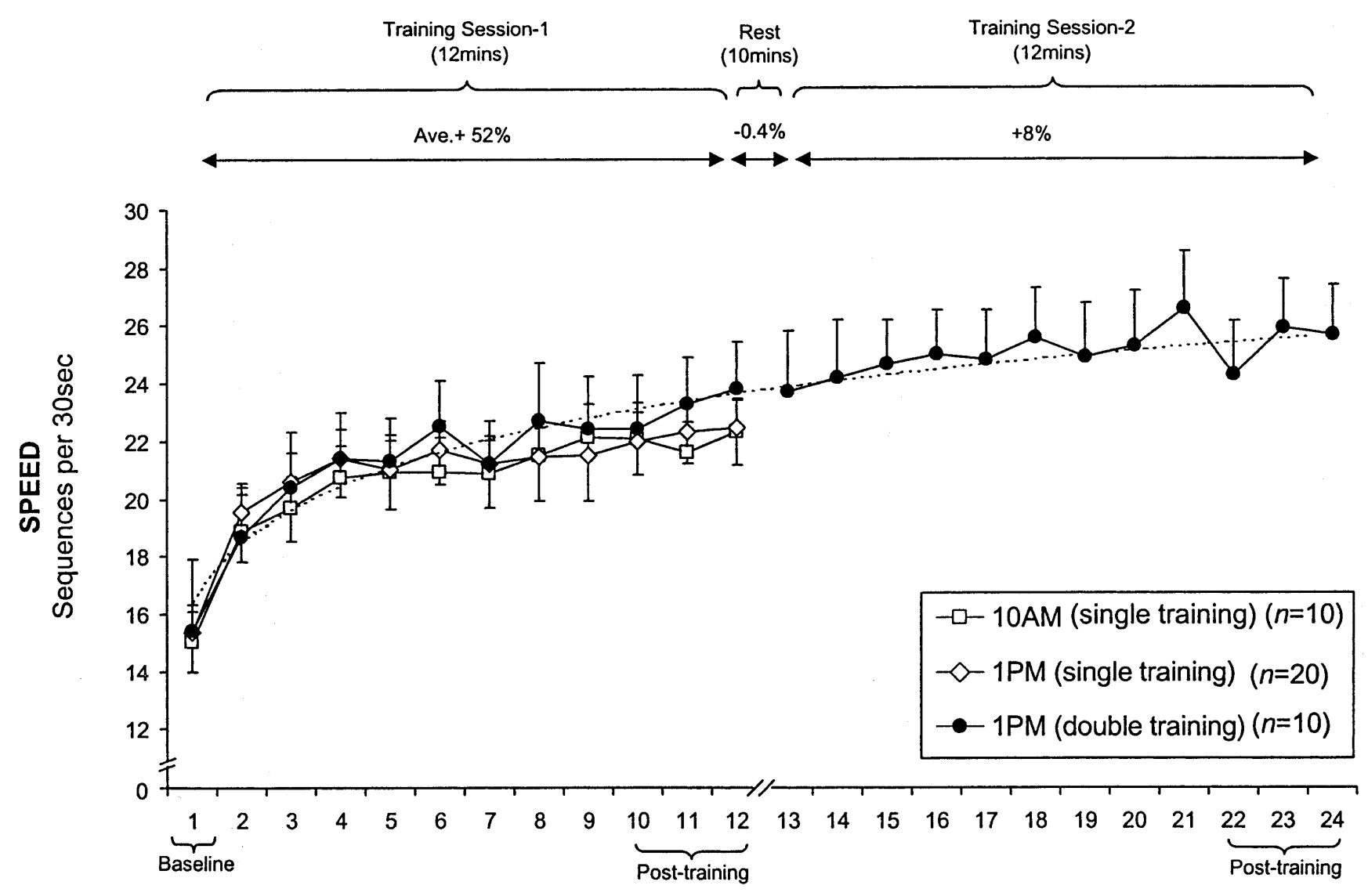

Training trial

Figure 1 Improvement in performance speed across training. Improvement during training session 1 (trials 1-12) was similar for subjects trained at 10 AM (group 1, $\square$ ) and 1 PM (groups 2 and 4, $\diamond$; and group 3, 0). Subjects in group 3 performed a second successive training session (trials $13-24$ ), demonstrating modest continued improvements, similar to the predicted improvement rate based on the logarithmic regression across the first 12 trials of training in Session 1 (broken line). 
difference in performance speed between the posttraining score (ave. trials $10-12)$ at $10 \mathrm{AM}$ or $1 \mathrm{PM}(\mathrm{t}[38]=0.19, P=0.84)$ or in the baseline starting level (trial $1 ; \mathrm{t}[38]=0.13, P=0.89$ ). When averaged across these groups, there was an improvement in performance speed of 7.8 sequences (52\%) during the 12 trials of training. The majority of this learning occurred across the first 3 trials ( 4.88 sequences; $32.4 \%$ or $1.6 \mathrm{seq} /$ trial), with the remaining 3.0 sequences $(19.7 \%)$ developing across the final 10 trials (or $0.33 \mathrm{seq} /$ trial; Fig. 1). On the basis of the learning curve across the 12 trials of Session One, a projected logarithmic fit, extending an additional 12 trials (13-24) could be extrapolated for additional tests to predict the expected performance-rate improvements following continued rehearsal (Fig. 1; broken line). There was also a significant $47 \%$ decrease in error rate across the 12 training trials of Session One (0.29 errors/seq during Trial 1 vs. $0.15 /$ seq during Trial 12; $\mathrm{t}[39]=2.61, P=0.01)$.

\section{Training Session 2}

Following a 10-min rest period after Session One, subjects in group 4 received a second training session (Session Two). Subjects showed small, but significant continued incremental increases in performance speed, improving by a total of just 2.0 sequences across the additional 12 trials, from 23.7 to 25.7 sequences ( $8.44 \%$ or 0.17 seq/trial, $\mathrm{r}=0.67, \mathrm{df}=11, P=0.01$; Fig. 1 ). As can be seen from Figure 1, the learning benefit achieved across Session Two was similar to the extrapolated/predicted improvement rate on the basis of the initial 12 trials across training Session One (Fig. 1, broken line). There was no significant difference between the predicted improvement based on the continued rehearsal rate of $0.33 \mathrm{seq} /$ trial during the final 10 trials of training in Session One, and the actual improvement obtained by subjects across Session Two $(t[9]=1.60, p=0.14)$. There was also no significant difference between performance on the final trial of Session One (Trial 12; $23.8 \mathrm{seq} /$ trial) and the first trial of Session Two (Trial 13; $23.7 \mathrm{seq} /$ trial) following the 10-min rest period ( $\mathrm{t}[9]=0.15, P=0.88)$. In contrast to the modest increase in speed, there was no significant reduction in error rate across Session Two (0.16 for Trial 13 vs. 0.14 for Trial 24 ; $\mathrm{t}[9]=0.53$, $P=0.60)$.

\section{Group 1: Motor Skill Learning Across $36 \mathrm{~h}(n=10)$}

To investigate differences in the profile of continued motor-skill learning across periods of wake prior to a night of sleep compared with periods of wake following a night of sleep, subjects in group 1 (Fig. 5, below) were training at $10 \mathrm{AM}$ day 1 , and retested at 2, 6 , and $10 \mathrm{PM}$ later that same day (elapsed time $=4-12 \mathrm{~h}$ ). Then,

Figure 2 Differential motor skill learning across $36 \mathrm{~h}$ before and after sleep (Group 1). (A) Speed. Following training at 10 AM, subjects demonstrated modest, but significant continued gains in motor-skill speed with rehearsal on day 1 (solid black bars). The initial retest on day 2 (RT4) showed a large and significant overnight improvement. In contrast, continued retesting across day 2 (hatched bars) yielded no further increase in performance speed. The marked difference in learning profiles prior to sleep (day 1 ) and following sleep (day 2 ) are seen in the improvement slopes for each group (broken lines; day 1 slope [presleep] $=1.08$ vs. day 2 slope [postsleep] $=0.14)$. (B) Error rate. In contrast, error rate did not significantly improve during daytime rehearsal on either day 1 (solid bars) or day 2 (hatched bars), with the only significant decrease in error rate occurring exclusively across the night of intervening sleep. This similarity is evident in the comparable learning profile slopes prior to sleep on day 1 and following sleep on day 2 (broken lines; day 1 slope [presleep] = 0.009 vs. day -2 slope $[$ postsleep] $=0.001)$. (PT) Posttraining value (average of final three trials of training); (RT) retest value (average of three trials); Error bars, SEM; (Asterisks) significance $(P)$ compared with previous time point at ${ }^{*}<0.05 ;{ }^{* *}<0.005$ following a night of sleep, subjects were retested again across the subsequent waking period of day 2 at $10 \mathrm{AM}, 2,6$, and $10 \mathrm{PM}$ (time $=24-36 \mathrm{~h})$.

\section{Speed}

There was a significant difference in performance speed across the 36 -h period $(\mathrm{F}[7,72]=2.59, P=0.01$; Fig. $2 \mathrm{~A})$. Following training, subjects demonstrated a significant linear increase in performance at each retest across the first $12 \mathrm{~h}$ of wake on day 1 (Fig. 2A, solid black bars; F[4,45] $=8.06, P<0.001$ ), improving by

\section{Group 1}

(A)

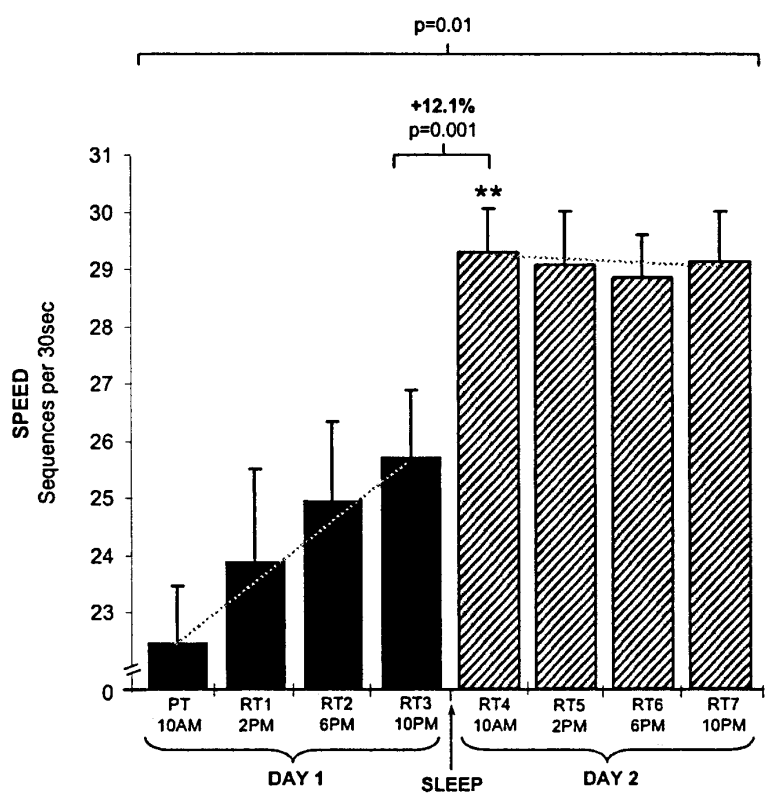

(B)

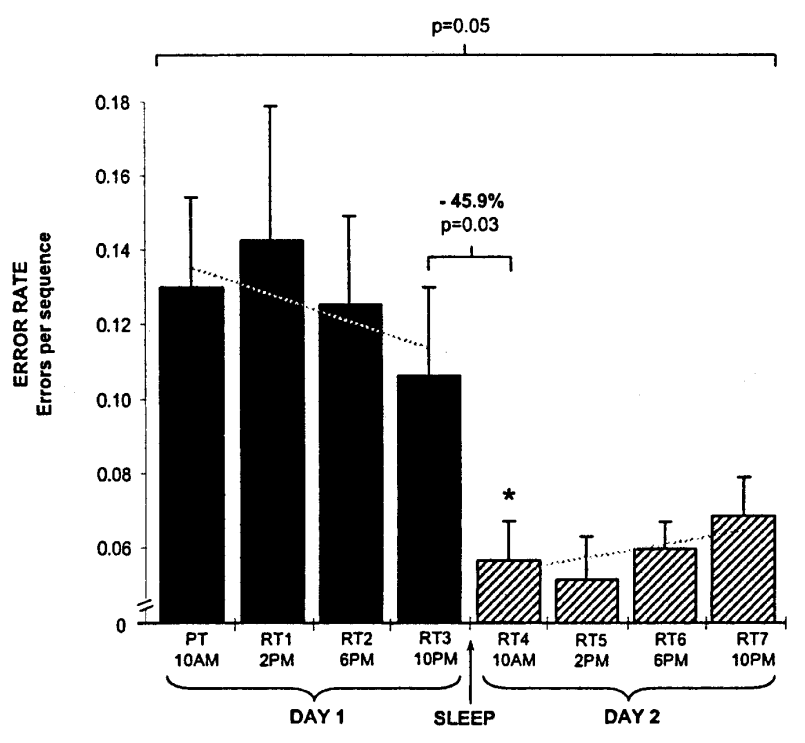


an average of 1.1 sequences per retest, or 0.37 seq/trial. These incremental gains in performance speed across day 1 were not significantly different from the expected rehearsal-based improvement extrapolated from the final 10 trials of training (paired t-tests, $t>0.18, P>0.4$ for each time, $t=1.3, P>0.2$ for all times combined). Thus, with continued retesting across day 1 prior to sleep, there was a modest, linear improvement in performance speed, similar to that predicted by rehearsal alone.

Although these subjects displayed large significant improvements overnight (see below), continued retesting across day 2 resulted in no additional performance gains at 2,6 , or 10 PM (Fig. 2A, hatched bars; F[3,36] $=0.01, P=0.99$ ). The differences in learning profiles prior to sleep (day 1) and following sleep (day 2) are demonstrated by the performance slopes for each day in Figure 2A. When the performance slopes across the four time points on day 1 and day 2 were compared (Fig. 2A; broken lines representing the linear approximation fit), there was a significant difference between the rate of improvement in performance on presleep day 1 compared with the postsleep performance profile on day $2(\mathrm{t}[9]=3.09, P=0.01)$ suggesting that, following the overnight gains in motor-skill speed, no subsequent rehearsal-dependent learning occurred on day 2 .

In agreement with our previous findings (see below), a comparison of the final retest performance prior to sleep on day 1 (RT3, 10 PM) with the first retest the next morning (RT4, $10 \mathrm{AM}$ ) revealed a significant increase in learning overnight $(\mathrm{t}[9]=4.33$, $P=0.001$; Fig. 2A).

\section{Error Rate}

There was also a significant difference in error rate across the $36-\mathrm{h}$ period $(\mathrm{F}[7,72]=2.10, P<0.05$; Fig. $2 \mathrm{~B})$. But contrary to the findings for speed improvement, there was no significant change in error rate across tests on day 1 (Fig. 2B, solid black bars; $\mathrm{F}[4,45]=1.19, P=0.32$ ) or following sleep, across day 2 (Fig. $2 \mathrm{~B}$, hatched bars; $\mathrm{F}[3,36]=0.28, P=0.82$ ). This was further confirmed by a comparison of the error rate slopes calculated across the four time points on days 1 and 2 (Fig. 2B; broken lines), which also did not differ significantly from each other $(\mathrm{t}[9]=1.2$, $P=0.25)$, or from zero for each day separately (t $[9]<1.1, P>0.28)$. However, a comparison of performance at the final retest prior to sleep on day 1 (RT3, 10 PM) with the first retest following sleep on day 2 (RT4, $10 \mathrm{AM}$ ) revealed a large and significant $45.9 \%$ reduction in the error rate expressly overnight $[\mathrm{t}(9)=2.54, P=0.03$; Fig. 2B]

In summary, sleep resulted in large overnight improvements in speed and equally large improvements in error rate. But whereas gains in motor skill speed were seen across daytime rehearsal on day 1 but not day 2 , there were no significant reductions in error rate across the waking interval on either day 1 or day 2.

\section{Group 2: Motor Skill Learning Across $24 \mathrm{hr}$ $(n=10)$}

Previously, we have demonstrated that a night of sleep resulted in an average $18 \%$ improvement in motor-skill speed following a single training session, shown in groups 5 and 6 (Fig. 3), whereas periods of ${ }^{* *}<0.005$ daytime wake offered no significant improvement beyond that expected from rehearsal alone (Walker et al. 2002). Furthermore, although error rates decreased by $\sim 35 \%$ overnight, they also showed no improvement across periods of wake (Fig. 3). For comparative purposes, we trained subjects in group 2 at $1 \mathrm{PM}$, and then retested them once $24 \mathrm{~h}$ later (rather than at 12-h intervals as in our previous study) to confirm that a similar degree of overnight motor skill learning was evident at 1 PM day $2(+24 \mathrm{~h})$. At the 24-h retest, following a single training session and one night of sleep, subjects' performance speed improved by

\section{Group 5}

(A)

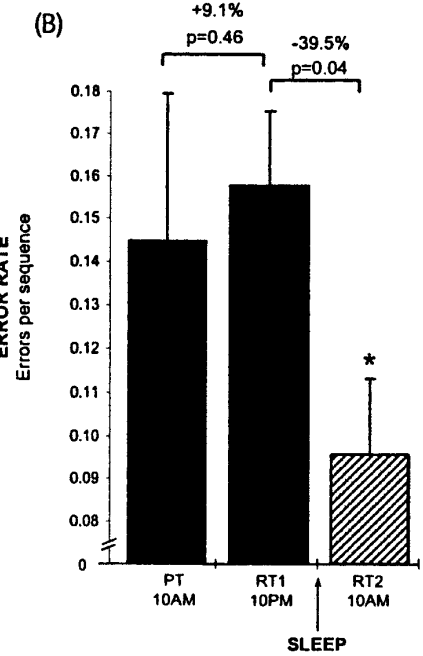

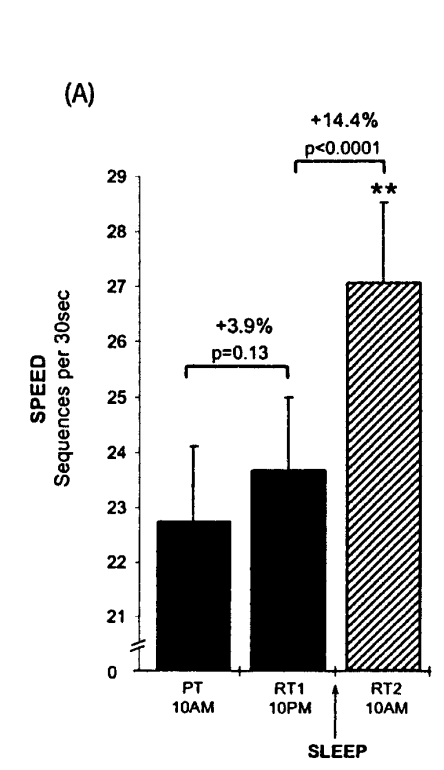

Group 6

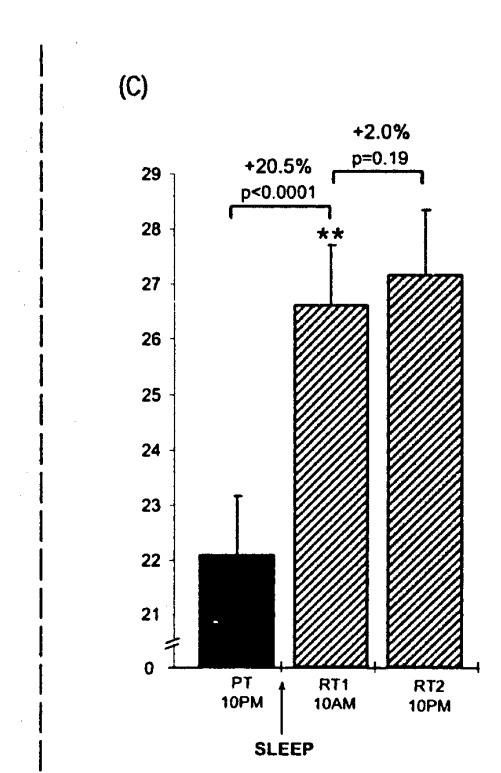

Figure 3 Continued motor-skill learning across $24 \mathrm{~h}$ with wake or sleep first (Groups 5 and 6, modified from Walker et al. 2002). (A,B) Group 5: Following a single training session in the morning, subjects demonstrated no significant change in speed or error rate following $12 \mathrm{~h}$ of wake (RT1; solid bars) relative to the end of training (posttraining; PT). However, following a night of sleep, a significant increase in speed and decrease in error rate had developed (RT2; hatched bar). (C,D) Group 6: Subjects were trained in the evening, and showed significant improvements in speed and error rate after only $12 \mathrm{~h}$, following a night of sleep (RT1, solid bar), but displayed no further significant improvements in speed or error rate with an additional $12 \mathrm{~h}$ of wake (RT2, hatched bars). Error bars, SEM; (Asterisks) significance $(P)$ compared with previous time point at ${ }^{*}<0.05$; 


\section{Group 2}

(A)
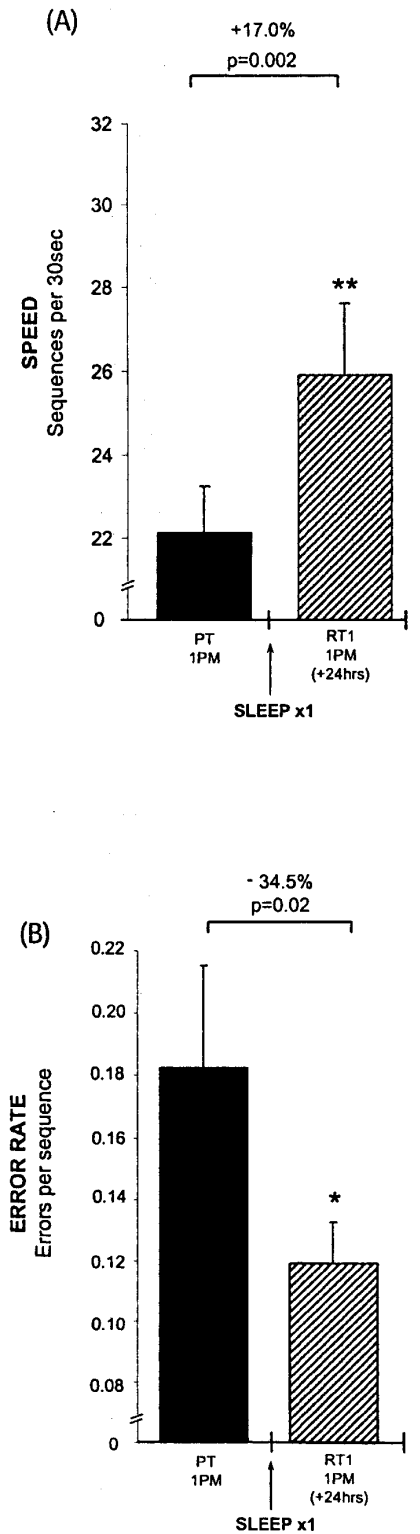

Group 3

(C)

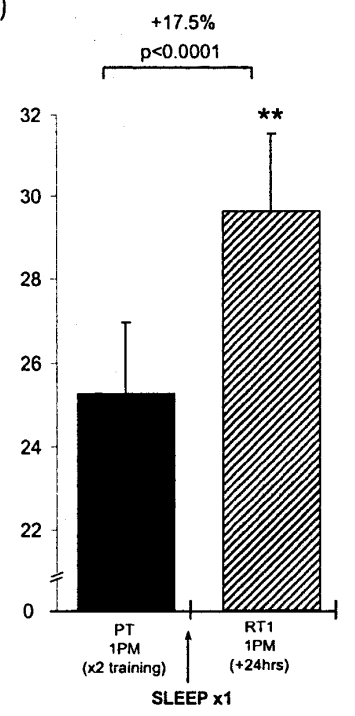

(D)

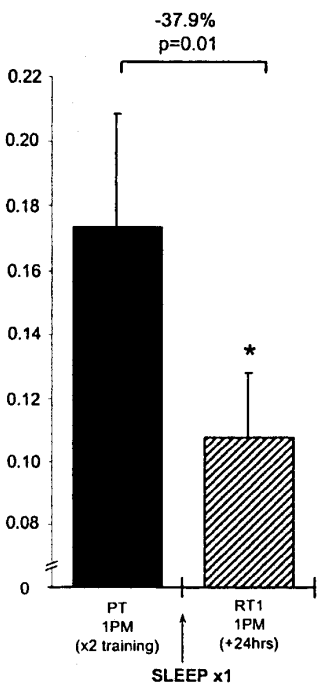

Group 4

(E)

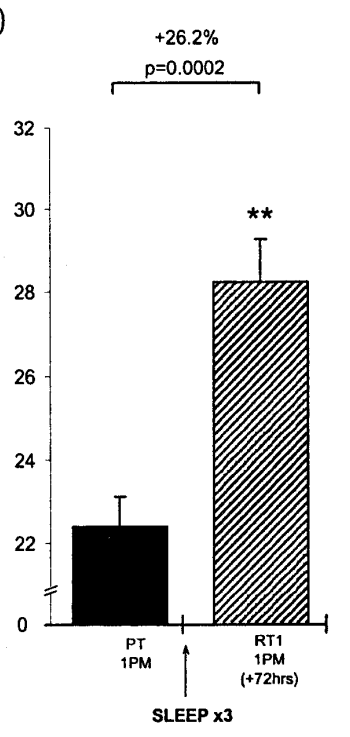

(F)

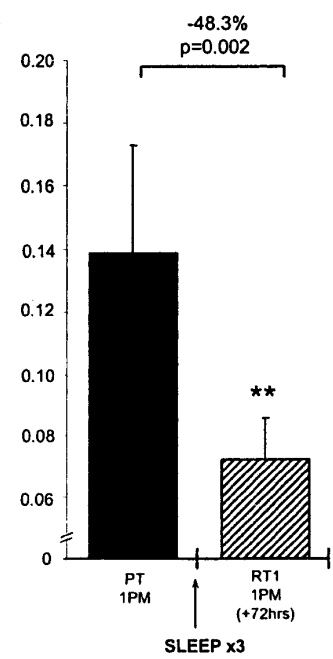

Figure 4 Continued motor-skill learning across $24-72 \mathrm{~h}$ with variable training and intervening nights of sleep (Groups 2-4). ( $A, B)$ Group 2: Subjects received a single training session on day $1(1 \mathrm{PM})$, and demonstrated significant increases in speed $(A)$ and decreases in error rate $(B)$ relative to the end of training (PT) when retested $24 \mathrm{~h}$ later on day 2 (1 PM; RT1), similar to overnight values seen in our earlier findings (see Fig. 3). (C,D) Group 3: Subjects performed two consecutive training sessions on day 1 (1 PM). Although subjects attained different posttraining (PT) performance levels relative to subjects in group 2 due to this second training session, they displayed almost identical overnight percentage improvements in speed (C) and reductions in error rate $(D)$ at retest on day $2(\mathrm{RT} 1)$. (E, F) Group 4: Subjects received a single training session as in group 2, but were instead retested $72 \mathrm{~h}$ later after three intervening nights of sleep, and showed larger increases in performance speed $(E)$ and greater reductions in error rate $(F)$. Error bars, SEM; (Asterisks) significance $(P)$ compared with previous time point at ${ }^{*}<0.05 ;{ }^{* *}<0.005$.

$17.0 \%$ (posttraining $=22.1 \mathrm{seq} /$ trial versus postsleep $=25.9 \mathrm{seq} /$ trial, $\mathrm{t}[9]=4.12, P=0.002$; Fig. $4 \mathrm{~A}$, top) as their error rate decreased by $34.5 \%$ (t $[9]=2.72, P=0.02$; Fig. 4 A, bottom).

Thus, the improvements in both speed and accuracy occurring across $24 \mathrm{~h}$ in group 2 were similar to those reported previously (Walker et al. 2002) across $12 \mathrm{~h}$, including a night of sleep in group 6 (speed: $\mathrm{t}[21]=1.10, P=0.28$; error rate, $\mathrm{t}[21]=1.17$, $\mathrm{P}=0.25)$.
Group 3: Motor Skill Learning Across 24 h Following a Double Training Session $(n=10)$

Subjects in group 3 were trained once at 1 PM day 1, but following a 10-min rest period, underwent a second training session of an additional 12 trials, and were then retested $24 \mathrm{~h}$ later, after one night of sleep. Relative to the end of their second training session, subjects in group 3 displayed an average $17.5 \%$ improve- 
ment in speed (posttraining $=25.3 \mathrm{seq} /$ trial vs. postsleep $=29.7$ seq/trial, $\mathrm{t}[9]=7.2, P<0.0001 ;$ Fig. $4 \mathrm{~B}$, top) and a $37.9 \%$ decrease in relative error rate $(\mathrm{t}[9]=2.89, P=0.01$; Fig. $4 \mathrm{~B}$, bottom). The overnight learning effects in group 3 were almost identical to those observed in group 2 following just one training session for both motor skill speed $(+17 \%$ vs. $+17.5 \%$; $\mathrm{t}[18]=0.28, P=0.77)$ and error rate $(-34.5 \%$ vs. $-37.9 \% ; \mathrm{t}[18]=0.72, P=0.48)$. Therefore, whereas doubling the intensity of initial training did allow some additional rehearsal-dependent improvements during Session 2, it did not appear to change the subsequent quantity of overnight sleep-dependent learning that developed.

Group 4: Motor Skill Learning Across 72 h $(n=10)$ Subjects in group 4 were trained only once (12 trials) at $1 \mathrm{PM}$ on day 1 as in group 2 . But instead of being retested $24 \mathrm{~h}$ later, following one night of sleep, they were retested $72 \mathrm{~h}$ later, following three nights of sleep, on day 4 . At the 72-h retest, subjects showed marked improvements in both performance speed $(+26.2 \%$; $\mathrm{t}[9]=6.20, P<0.0001$; Fig. $4 \mathrm{C}$, top) and error rate $(-48.3 \%$; $[9]=2.58, P=0.002$; Fig. $4 \mathrm{C}$, bottom). Improvements seen after three nights of sleep showed trends toward being greater than those seen after one night of sleep in group 2 for both speed $(+26.2 \%$ vs. $+17.0 \% ; \mathrm{t}[18]=1.96, P=0.07)$ and error rate $(-48.3 \%$ vs. $-34.5 \% ; \mathrm{t}[18]=1.68, P=0.10)$. This trend toward greater speed after three nights of sleep was seen even when compared with subjects who received two training sessions rather than one and one night of sleep (group 3; speed: $\mathrm{t}[18]=2.0, P=0.06)$.

\section{Training-Dependent Learning Versus Sleep-Dependent Learning}

Whereas two stages of motor-skill learning were apparent; one initial rapid learning stage during training, and a second phase developing across a night of sleep, an outstanding question remained-does the amount of training-dependent learning during acquisition (i.e., learning during practice) influence or show any relationship with the amount of sleep-dependent learning that individuals subsequently achieve? To investigate this possibility, the percentage of improvement in motor-skill learning across training (difference between baseline score and posttraining score) and the percentage of delayed sleep-dependent improvement (difference between the posttraining score and the retest score following a night of sleep) was correlated in the 25 subjects who were trained once on day 1 and retested once after a night of sleep on day 2 (groups 2 and 6 combined). Surprisingly, there was no evidence of a relationship between the efficiency of within session, training-dependent learning, and the amount of subsequent overnight sleep-dependent learning (practice-independent) with regards to performance speed $(\mathrm{r}=0.18$, $P=0.38$; Fig. 5A). Similarly, there was also no sign of a correlation between decreases in error rate within the training session compared with the reduction in error rate overnight $(\mathrm{r}=0.03$, $P=0.89$; Fig. 5B). Similarly, no relationship between the two learning stages was evident within each of the remaining four groups analyzed separately (speed: $\mathrm{r}<0.35, P<0.18$; error rate: $<0.37, P<0.17$ for each group) or for all groups combined (speed: $\mathrm{r}=0.07, P=0.56$; error rate: $\mathrm{r}=0.13, P=0.26$ ).

One possible explanation for this lack of relationship is that both performance speed and error rate had simply reached asymptotic levels at the end of training and there was no possibility for a correlative match with the delayed overnight learning. However, two further analyses demonstrated this not to be the case. Firstly, there was still no correlation between the actual posttraining values (rather than percent improvement across training) and the amount of subsequent delayed sleep-dependent
(A)

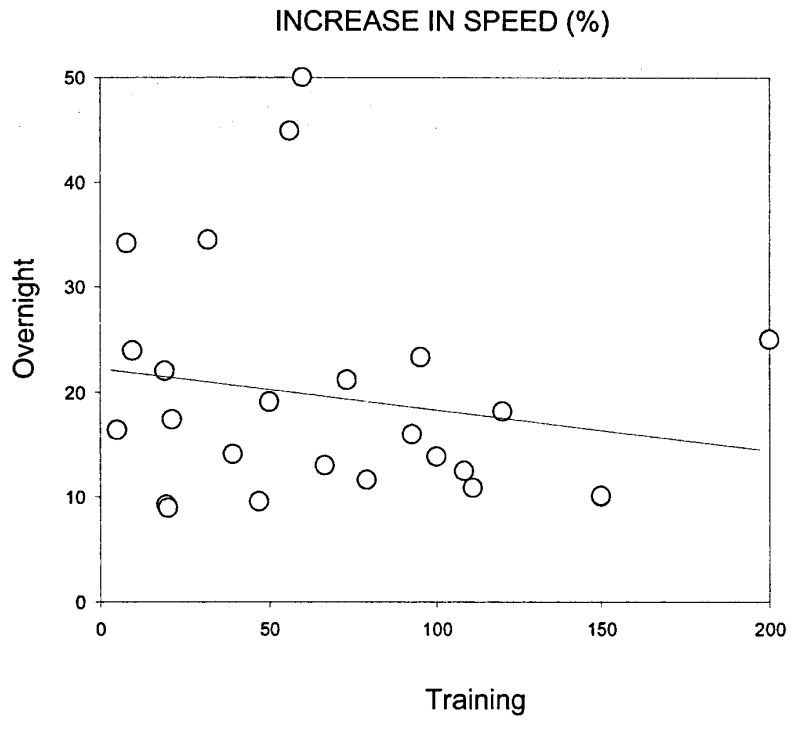

(B)

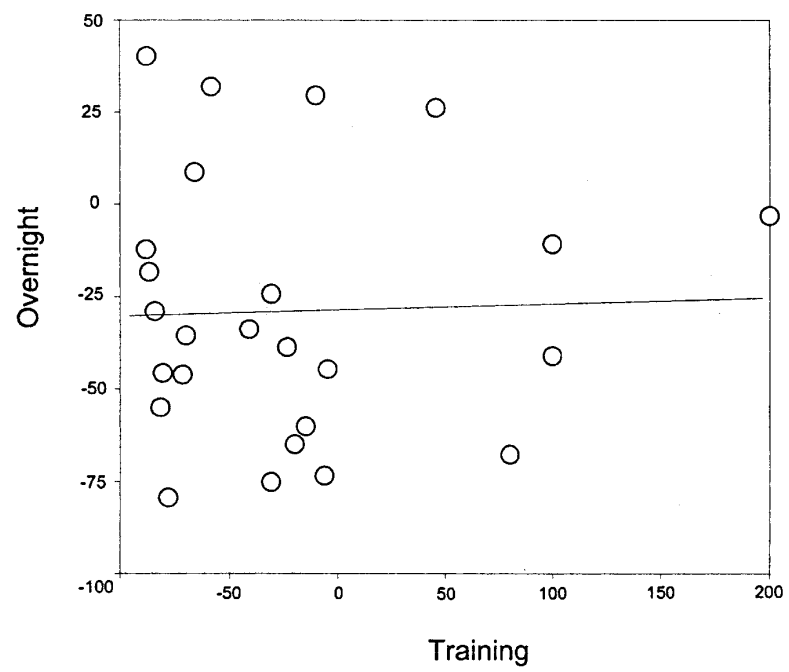

Figure 5 Training-dependent learning vs. sleep-dependent learning. Subjects were trained once and retested once after a single intervening night of sleep ( $n=25$; groups 2 and 5). (A) Speed: There was no relationship between the amount of improvement during training (difference between baseline and posttraining score) and the amount of subsequent overnight improvements (difference between posttraining and retest score). (B) Error rate: Similarly, there was no evidence of a correlation between practice-dependent changes in error rate during training, and the amount of continued performance improvements in error rate that developed overnight.

learning (speed: $\mathrm{r}=0.19, P=0.19$; error rate: $\mathrm{r}=0.22, P=0.14$ ). Secondly, when the 25 subjects were divided into 2 groups on the basis of posttraining mean score for either speed $(\leq 22>$ seq/trial) or error rate $(\leq 0.12>$ errors/seq), there was no significant difference between the upper and lower achievement groups in the amount of subsequent overnight delayed improvement that developed (speed: $\mathrm{t}[23]=0.79, P=0.43$; error rate: $\mathrm{t}[23]=1.61$, $P=0.12)$. 
Thus, the lack of correlation between practice-dependent learning and sleep-dependent learning did not appear to be due to posttraining asymptotic levels, and regardless of the performance ability achieved at the end of training, high or low, both groups appeared to develop an equal distribution of overnight sleep-dependent improvement, unrelated to the prior posttraining ability. Taken together, the lack of correlation suggests that the two stages of motor skill learning, one training dependent and the other sleep dependent, are distinct and independent processes.

\section{DISCUSSION}

Differential Motor-Skill Learning Before and After Sleep Subjects in group 1 were tested repeatedly on the motor-skill task across two consecutive days ( $36 \mathrm{~h}$ ) separated by an intervening night of sleep. Following a single training session in the morning, subjects showed the ability for small but continued incremental improvements in speed at each of the retest sessions across the first $12 \mathrm{~h}$ on day 1 prior to sleep, similar to that expected by continued task rehearsal, and without evidence of circadian fluctuations (Fig. 2A; solid bars). When retested immediately after the night of sleep, subjects showed a much larger improvement, far in excess of that predicted by additional rehearsal and similar to the overnight sleep-dependent improvements seen in our previous study (Fig. 3; Walker et al. 2002). However, following these sleep-dependent performance gains, the capacity for additional rehearsal-based learning appeared to temporarily stall, with continued retesting (rehearsal) across day 2 yielding no further improvements in motor-skill speed (Fig. 2A, hatched bars).

It would therefore appear that the large increase in performance produced by a night of sleep comes at the expense of potential improvement with rehearsal the next day. But, as discussed below, this diminished learning potential does not represent the end of all further improvement. A previous report has demonstrated that performance on this task continues to improve with repeat testing across many weeks (termed the slow component of learning), with subjects achieving greater than 35 seq/trial by week 5 before nearing asymptotic levels (Karni et al. 1998), far in excess of values achieved by subjects in this study.

Interestingly, error rate scores show a different profile across $36 \mathrm{~h}$. In contrast to motor skill speed, no significant change in error rate was seen with repeated testing across either day 1 or day 2 . The only reduction in error rate seen after the initial training session occurred across the intervening night of sleep. This is perhaps surprising considering that, despite the continued task rehearsals during each brief retest, periods of daytime wake still offered no consistent decreases in error rate, regardless of whether the waking episode came before or after a night of sleep.

Although not specifically designed to investigate circadian effects on motor skill learning, we reiterate that circadian influences are not likely to be the explanatory factor determining changes in motor learning observed across the 36-h period, for several reasons. Firstly, the profile of performance speed across day 1 is dramatically different from that of day 2 , despite being tested at identical time points on the circadian cycle. Similarly, the profile of error rate change across day 1 is completely reversed on day 2 , even though test times were identical on both days. Secondly, the initial learning curves across training were essentially identical for subjects trained in the morning, afternoon, and evening. Thirdly, the delayed, sleep-dependent learning effect appears to occur only across periods of sleep, regardless of whether those sleep epochs occur during the day or during the night (Fischer et al. 2002; Walker et al. 2002). Finally, there was no significant difference in subjective ratings of alertness across all testing points. Thus, the changes in learning profile do not appear to be easily explained by any known profile of circadian rhythm, although some subtle influences may yet be identified.

It is therefore clear that a delayed, sleep-dependent learning effect is common for both motor-skill speed and error rate. Nevertheless, a subtle difference exists. Small practice-dependent improvements in performance speed, but not in error rate, are possible during brief retests before the occurrence of sleep, although following a night of sleep, further rehearsal offers no additional improvement in either speed or error rate.

The small, continued rehearsal-based improvements in motor-skill learning with repeat testing across the first day are different from a recent report of actual diminishing performance on a visual skill task with repeat daytime testing, the effects of which can be ameliorated with a brief period of daytime sleep (Mednick et al. 2002). Whereas the cause of this difference remains unclear, we consider several potential explanations. Firstly, the duration of retesting on these tasks is dramatically different. Retesting on the motor task in this study lasted just $3 \mathrm{~min}$, whereas the duration of each retest for the visual skill task was $>1 \mathrm{~h}$. Such prolonged durations of testing on the visual skill task may therefore trigger performance deteriorations across the day. It remains to be seen if short retest periods on the visual task provoke a similar profile of deterioration. Secondly, these task differences may also reflect a distinction between the input (sensory/perceptual) and output (motor) roles of each of the respective systems used. From a functional perspective, it is conceivable that the motor system simply does not fatigue as easily as the visual system.

\section{Sleep-Dependent Learning Following One or Two Training Sessions}

Another question regarding the sleep-dependent nature of motor-skill learning concerned the amount of initial training during acquisition. Subjects in group 2 received only one training session on day 1 and were retested $24 \mathrm{~h}$ later after a single night of sleep. When subjects in group 3 received a second training session on day 1 (10 min after completion of the first session), relatively modest increases in motor-skill speed and small reductions in error rate continued to develop. Interestingly, this further improvement did not diminish the amount of subsequent overnight improvement that developed, but neither did it increase the sleep-dependent learning effect.

The nearly identical percentages of overnight improvement in these two groups (speed: $+17.0 \%$ vs. $+17.5 \%$; error rate: $-34.5 \%$ vs. $-37.9 \%$ ) suggest that the strength or efficiency of memory representation formed after one training session is sufficient to maximize subsequent sleep-dependent modification, and although a second training session provides some additional performance gains within that practice session, it does not alter the amount of delayed overnight learning that will subsequently develop.

\section{Sleep-Dependent Learning Across Three Nights of Sleep Versus One Night of Sleep}

Although the extent of overnight improvement did not differ after one or two training sessions, additional nights of posttraining sleep do appear to confer additional learning benefits. Subjects in group 4 received a single training session on day 1 , but instead of being retested after only one night of sleep (as in group 2 ), were retested $72 \mathrm{~h}$ later, after three nights of sleep, and demonstrated large significant improvements in speed $(+26.2 \%)$ and error rate $(-48.3 \%)$.

Thus, whereas sleep on the first night following training offered the most dramatic learning benefit, subsequent nights of sleep provided more modest but continued gains. It is important to note that neither rehearsal nor the simple passage of wake 
time on day 2 led to any increase in speed. Thus, the subsequent improvement over additional days and nights would appear to again reflect sleep-dependent processes, and emphasize the robust and long-acting role that sleep appears to offer, not only for the maintenance of performance levels, but for the actual enhancement of learning.

It is of note that the temporal evolution of large gains in motor-skill learning immediately after the first night of sleep, followed by more modest continued improvements across subsequent nights of sleep, are similar to those reported for the time course of sleep-dependent visual skill learning (Stickgold et al. 2000a). The similarity of overnight gains resulting from multiple nights of sleep for these different perceptual and motor tasks may indicate a common underlying profile of sleep-dependent memory formation within the procedural memory domain.

\section{Training-Dependent Learning Versus \\ Sleep-Dependent Learning}

In our previous study, we found that the amount of stage-II NREM sleep, particularly in the last quarter of the night, could explain more than half of the variance in overnight improvement on the motor skill task (Walker et al. 2002). Another obvious factor that could determine the quantity of delayed learning is the amount of improvement achieved within the training session. However, although subjects all improved substantially across the training session, there was no correlation between the amount of learning achieved during training and the amount of subsequent sleep-dependent improvement, regardless of the posttraining level that individual subjects achieved. These correlations would suggest that the sleep-dependent process of improvement is unrelated to the practice-dependent process of learning during waking, reflecting two discrete stages of procedural memory formation, one developing rapidly during task performance and one developing across a night of sleep without active task engagement.

Although remaining speculative, it is interesting to note that this two-stage learning progression has intriguing parallels with cellular data regarding motor-skill learning. Rapid changes in neuronal responsiveness within the motor system involving the disinhibition or unmasking of latent intracortical connections (Jacobs and Donoghue 1991; Butefisch et al. 2000) have been demonstrated across the time frame of minutes, and may therefore be distinct from more slowly developing changes across hours or days that involve alterations in neuronal structure (e.g., synaptic connectivity) and function (e.g., synaptic sensitivity) (Soderling and Derkach 2000; Abel and Lattal 2001). It is tantalizing to suggest that a similar mechanistic difference underlies the independence seen between the rapid, within-session performance changes on our finger-tapping task and the more slowly developing, sleep-dependent changes that develop overnight.

In summary, it would appear firstly that the overnight motor-skill learning that occurs during sleep alters the capacity for

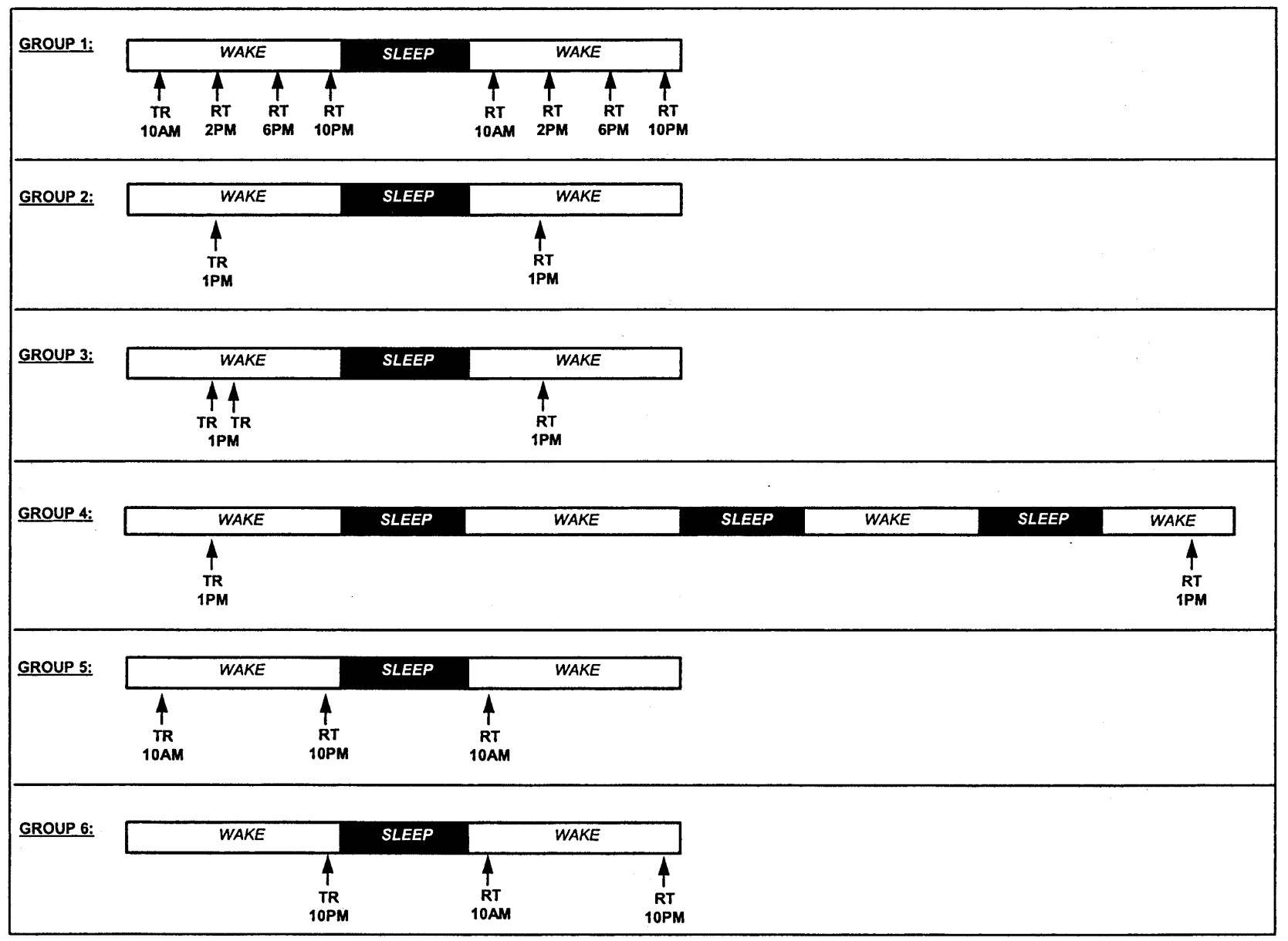

Figure 6 Experimental protocols. Forty subjects (groups 1-4) were trained at either 10 AM or 1 PM on day 1, and then retested across the following 24-72-h period in different experimental protocols. Thirty additional subjects (groups 5 and 6) were trained and retested in an earlier study (Walker et al. 2002) and are presented for comparison. (TR) Training; (RT) retest

\section{Learning \& Memory}

www.learnmem.org 
subsequent rehearsal-based improvements during waking episodes, so that prior to a night of sleep, small practice-dependent gains are possible, but following a night of sleep, this capacity is diminished. Secondly, doubling the duration of training does not appear to alter the amount of subsequent sleep-dependent learning. Furthermore, the amount of sleep-dependent learning does not correlate with the amount of practice-dependent learning during training, suggesting the existence of two discrete motorlearning processes. Finally, whereas the majority of sleep-dependent motor-skill learning appears to occur during the first night of sleep following training, additional nights of sleep appear to support continued improvements.

\section{MATERIALS AND METHODS}

\section{Participants}

The study cohort consisted of 40 right-handed subjects between the ages of 18 and 28 (mean age $24.3 \pm 2.1$ [SD]; 23 females). Subjects had no prior history of drug or alcohol abuse, neurological, psychiatric, or sleep disorders, and were instructed to be drug, alcohol, and caffeine free for $24 \mathrm{~h}$ prior to and during the study period. All studies were approved by the local human studies committee and all subjects provided written informed consent.

\section{Procedural Motor-Skill Task}

The sequential finger-tapping task required subjects to press four numeric keys on a standard computer keyboard with the fingers of their left (nondominant) hand, repeating the five-element sequence, 4-1-3-2-4, as quickly and as accurately as possible, for a period of $30 \mathrm{sec}$. The numeric sequence (4-1-3-2-4) was displayed at the top of the screen at all times to exclude any working memory component to the task. Each key press produced a white dot on the screen, forming a row from left to right and back, rather than the numbers typed, so as not to provide accuracy feedback. The computer recorded the key press responses, and each 30-sec trial was scored for the number of complete sequences achieved, designated as speed, and the number of errors made relative to the number of sequences (errors/sequence), designated as error rate. One single training session consisted of 12 30 -sec trials with 30 -sec rest periods between trials, and lasted a total of $12 \mathrm{~min}$. The scores (speed and error rate) from the first trial of the training session were taken as the baseline measure, whereas the averaged scores from the final three trials were defined as the posttraining performance. At each subsequent retest, subjects performed three 30 -sec trials of the same sequence separated by 30 -sec rest periods, with the scores again being averaged.

\section{Experimental Groups}

The 40 subjects were divided into 4 experimental groups of 10 subjects each, and were trained at either 10 AM or 1 PM Day 1. Each group subsequently underwent a specific schedule of retests. All training and test sessions were performed within $30 \mathrm{~min}$ of the times indicated, and morning retests were performed at least $1 \mathrm{~h}$ after awakening. The testing schedules, shown in Figure 6, were as follows:

\section{Group 1: Motor-Skill Learning Across $36 h(n=10)$}

Subjects received one training session (12 trials) at 10 AM day 1 , and were retested at 4-h intervals across the rest of day 1 (4-12 $\mathrm{h}$ posttraining), and then retested, again at 4-h intervals on day 2 (24-36 $\mathrm{h}$ posttraining) following a night of sleep.

\section{Group 2: Motor-Skill Learning Across $24 h(n=10)$}

Subjects received one training session (12 trials) at 1 PM on day 1 and were retested once, $24 \mathrm{~h}$ later (1 PM, day 2$)$, following one night of intervening sleep. This group served primarily as a control for groups 3 and 4.
Group 3: Motor-Skill Learning Across 24 h Following a Double Training Session $(n=10)$

Subjects received two training sessions $(2 \times 12$ trials), separated by a 10 -min rest, at $1 \mathrm{PM}$ on day 1 , and were retested $24 \mathrm{~h}$ later (1 PM, day 2) following one night of intervening sleep.

Group 4: Motor-Skill Learning Across $72 h(n=10)$

Subjects received one training session (12 trials) at $1 \mathrm{PM}$ on day 1 , and were retested $72 \mathrm{~h}$ later (1 PM, Day 4), following three nights of intervening sleep.

We also describe relevant data from our previous study (see Walker et al. 2002 for details). These data are for two groups of subjects.

\section{Group 5: Motor-Skill Learning Across 24 h, With Wake First $(n=15)$}

Subjects received one training session (12 trials) at 10 AM on day 1 and were retested ( 2 trials) $12 \mathrm{~h}$ later that same day (10 PM) and again $24 \mathrm{~h}$ after training (10 AM, day 2), after a night of sleep.

Group 6: Motor-Skill Learning Across 24 h, With Sleep First $(n=15)$ Subjects received one training session (12 trials) at 10 PM on day 1 and retested ( 2 trials) 12 hrs later (10 AM, day 2) after a night of sleep, and again $24 \mathrm{~h}$ after training (10 PM, day 2).

With these additional 30 subjects, data are reported for a total of 70 subjects.

\section{Sleep Quality and Alertness}

At each training and retesting point, all subjects completed the Stanford Sleepiness Scale, a standard measure of subjective alertness (Hoddes et al. 1973). There were no significant differences in the Stanford Sleepiness Scale ratings of alertness within any of the groups across test points $(P \geq 0.35$ for each). On the 7 -point scale ( 1 being most alert), mean values were as follows: $10 \mathrm{AM}-$ 2.48, 1 PM-1.89, 2 PM-2.20, 6 PM-2.33, 10 PM-2.32.

The amount of overnight sleep obtained by subjects in each group was documented with sleep logs, and averaged $7.82 \pm 0.72 \mathrm{~h}(\mathrm{SD})$ of sleep across experimental nights.

\section{Statistical Analysis}

Analyses were carried out with one-way and two-way repeated measures ANOVA's and paired and unpaired two-tailed Students t-tests. All correlations between experimental variables were performed using Pearson's correlation analyses.

\section{ACKNOWLEDGMENTS}

We thank Mercedes Antienza, Jose Cantero, Ed Pace-Schott, Bernat Kocsis, and Roar Fosse for their helpful and constructive comments regarding the study. This research was supported by National Science Foundation grant BCS-0121953 (M.P.W.) and NIH grants MH-48,832 and DA-11,744 (J.A.H.).

The publication costs of this article were defrayed in part by payment of page charges. This article must therefore be hereby marked "advertisement" in accordance with 18 USC section 1734 solely to indicate this fact.

\section{REFERENCES}

Abel, T. and Lattal, K.M. 2001. Molecular mechanisms of memory acquisition, consolidation and retrieval. Curr. Opin. Neurobiol. 11: $180-187$.

Brashers-Krug, T., Shadmehr, R., and Bizzi, E. 1996. Consolidation in human motor memory. Nature 382: 252-255.

Butefisch, C.M., Davis, B.C., Wise, S.P., Sawaki, L., Kopylev, L., Classen, J., and Cohen, L.G. 2000. Mechanisms of use-dependent plasticity in the human motor cortex. Proc. Natl. Acad. Sci. 97: 3661-3665.

Fischer, S., Hallschmid, M., Elsner, A.L., and Born, J. 2002. Sleep forms memory for finger skills. Proc. Natl. Acad. Sci. 99: 11987-11991.

Gais, S., Plihal, W., Wagner, U., and Born, J. 2000. Early sleep triggers memory for early visual discrimination skills. Nat. Neurosci. 3: $1335-1339$.

Hoddes, E., Zarcone, V., Smythe, H., Philips, R., and Dement, W.C. 1973. Quantification of sleepiness: A new approach. Psychophysiology 10: $431-436$. 
Walker et al.

Jacobs, K.M. and Donoghue, J.P. 1991. Reshaping the cortical motor map by unmasking latent intracortical connections. Science 251: 944-947.

Jancke, L., Gaab, N., Wustenberg, T., Scheich, H., and Heinze, H.J. 2001. Short-term functional plasticity in the human auditory cortex: An fMRI study. Brain Res. Cogn. Brain Res. 12: 479-485.

Karni, A. and Bertini, G. 1997. Learning perceptual skills: Behavioral probes into adult cortical plasticity. Curr. Opin. Neurobiol. 7: 530-535.

Karni, A., Tanne, D., Rubenstein, B.S., Askenasy, J.J., and Sagi, D. 1994 Dependence on REM sleep of overnight improvement of a perceptual skill. Science 265: 679-682.

Karni, A., Meyer, G., Jezzard, P., Adams, M.M., Turner, R., and Ungerleider, L.G. 1995. Functional MRI evidence for adult motor cortex plasticity during motor skill learning. Nature 377: 155-158.

Karni, A., Meyer, G., Rey-Hipolito, C., Jezzard, P., Adams, M.M., Turner, R., and Ungerleider, L.G. 1998. The acquisition of skilled motor performance: Fast and slow experience-driven changes in primary motor cortex. Proc. Natl. Acad. Sci. 95: 861-868.

McGaugh, J.L. 2000. Memory-A century of consolidation. Science 287: $248-251$.
Mednick, S.C., Nakayama, K., Cantero, J.L., Atienza, M., Levin, A.A., Pathak, N., and Stickgold, R. 2002. The restorative effect of naps on perceptual deterioration. Nat. Neurosci. 5: 677-681.

Rattoni, F.B. and Escobar, M. 2000. Neurobiology of learning. In International handbook of psychology (ed. K. Pawlik and M. Rosenzweig), Vol. xxxii. Sage Publications Ltd., London, UK.

Soderling, T.R. and Derkach, V.A. 2000. Postsynaptic protein phosphorylation and LTP. Trends Neurosci. 23: 75-80.

Stickgold, R., James, L., and Hobson, J.A. 2000a. Visual discrimination learning requires sleep after training. Nat. Neurosci. 3: 1237-1238.

Stickgold, R., Whidbee, D., Schirmer, B., Patel, V., and Hobson, J.A. 2000b. Visual discrimination task improvement: A multi-step process occurring during sleep. J. Cogn. Neurosci. 12: 246-254.

Walker, M.P., Brakefield, T., Morgan, A., Hobson, J.A., and Stickgold, R. 2002. Practice with sleep makes perfect: Sleep dependent motor skill learning. Neuron 35: 205-211.

Received January 6, 2003; accepted in revised form May 21, 2003. 


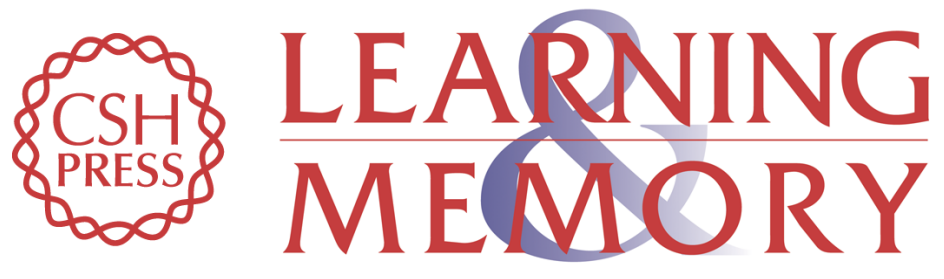

\section{Sleep and the Time Course of Motor Skill Learning}

Matthew P. Walker, Tiffany Brakefield, Joshua Seidman, et al.

Learn. Mem. 2003, 10:

Access the most recent version at doi:10.1101/lm.58503

References This article cites 18 articles, 6 of which can be accessed free at: http://learnmem.cshlp.org/content/10/4/275.full.html\#ref-list-1

License

Email Alerting Receive free email alerts when new articles cite this article - sign up in the box at the Service top right corner of the article or click here. 